\title{
Long term effect of intravenous immunoglobulins and oral cyclophosphamide in multifocal motor neuropathy
}

Nicoletta Meucci, Alberto Cappellari, Sergio Barbieri, Guglielmo Scarlato, Eduardo Nobile-Orazio

\begin{abstract}
Objectives-To report the long term effect of the combined treatment with high dose intravenous immunoglobulins (IVIg) and oral cyclophosphamide (CTX) in patients with multifocal motor neuropathy, and to determine whether the association of oral CTX in these patients may help to delay and, possibly, suspend IVIg infusions.

Methods-Six patients with multifocal motor neuropathy responding to an initial course of IVIg $(0.4 \mathrm{~g} / \mathrm{kg} / \mathrm{day}$ for five consecutive days) were followed up for 37 to 61 (mean 47) months. All patients were subsequently treated with periodic IVIg infusions $(0.4 \mathrm{~g} / \mathrm{kg} / \mathrm{day}$ for two days at clinical worsening) and oral CTX (1-3 $\mathrm{mg} / \mathrm{kg} / \mathrm{day})$. Improvement was assessed using the Rankin disability scale, a functional impairment scale for upper and lower limbs, and the MRC rating scale on the 20 most affected muscles. Electrophysiological and antiglycolipid antibody studies were performed before treatment, then yearly during follow up.
\end{abstract}

Results-All patients improved during treatment and, by the end of follow up or before worsening after therapy suspension, the median Rankin $(P=0.0335)$ and upper $(P=0.0015)$ and lower limb $(\mathbf{P}=\mathbf{0 . 0 3 0 1})$ impairment scores and the mean MRC $(P=0.0561)$ score were improved. By that time the number of nerves with partial motor conduction block was reduced $(P=0.0197)$ and antiglycolipid antibody titres had decreased in all but one patient. All patients required periodic IVIg infusions to maintain improvement but, after three to seven months of oral CTX, the interval between IVIg infusions could be progressively prolonged until, in three patients, both treatments could be stopped for up to two years before clinical worsening. The main complications, both related to oral CTX, were haemorrhagic cystitis in two patients and persistent amenorrhea in one patient.

Conclusions-IVIg can induce and maintain improvement in multifocal motor neuropathy but does not eradicate the disease. Oral CTX may help to induce a sustained remission but it is not devoid of side effects and might therefore be reserved for patients with multifocal motor neuropathy who require frequent IVIg infusions to maintain improvement.

(F Neurol Neurosurg Psychiatry 1997;63:765-769)

Keywords: IVIg; cyclophosphamide; multifocal motor neuropathy; therapy; antiglycolipid antibodies

Multifocal motor neuropathy is a chronic, asymmetric, motor neuropathy electrophysiologically characterised by the presence of persistent partial conduction block in motor but not sensory nerves. ${ }^{1-7}$ The pathogenesis of multifocal motor neuropathy is not known but there is some evidence, based mostly on the clinical improvement after immunological therapies $^{7-13}$ and the frequent detection of antiglycolipid antibodies in patients' serum, ${ }^{4-12}$ that the disease may have an immunological basis. High dose intravenous immunoglobulin therapy (IVIg) is rapidly effective in most patients, ${ }^{9-14}$ but its effects are transient and improvement has to be maintained with periodic IVIg infusions, ${ }^{15-17}$ rendering it a very expensive treatment. Among immunosuppressive agents, only high dose intravenous cyclophosphamide (CTX) is consistently effective in multifocal motor neuropathy, ${ }^{5-8}$ but its side effects $^{1819}$ may not warrant its use in less severely affected patients, especially if they are young. We previously reported that the addition of low dose oral CTX to IVIg was well tolerated by patients with multifocal motor neuropathy and that it permitted them to progressively reduce the frequency of IVIg infusions. ${ }^{9}$ Little is known, however, about the long term effect of this combined therapy in multifocal motor neuropathy.

\section{Methods}

PATIENTS

Six patients with multifocal motor neuropathy, who responded to an initial five day course of IVIg at a daily dose of $0.4 \mathrm{~g} / \mathrm{kg}$, were studied. The clinical and electrophysiological features of three of them (patients 1,2, and 3) including their initial response to IVIg have been previously reported. ${ }^{9}$ In all patients the diagnosis of multifocal motor neuropathy was based on the presence of progressive, asymmetric limb weakness with or without minimal sensory impairment, who showed persistent partial conduction block in at least two motor nerves but not in sensory nerves. Partial motor conduction block was defined as a reduction in and in final revised form 12 June 1997

Accepted 18 June 1997 
the ratio of proximal to distal CMAP amplitude to $<0.60$ (corresponding to a $40 \%$ partial motor conduction block) when the ratio of proximal to distal negative peak duration was $<1.15$. $^{9}$

TREATMENT REGIMEN

After the initial five day course of IVIg (Sandoglobulin; Sandoz; Basel, Switzerland, or Ig Vena; Sclavo; Siena, Italy)(repeated again in patients 1,2, and 3 after two months), patients were treated with two day IVIg infusions (0.4 $\mathrm{g} / \mathrm{kg}$ /day) at the time of clinical worsening. Oral CTX was added at a daily dose of $1.5-2 \mathrm{mg} / \mathrm{kg}$ after the first IVIg course (patients 4, 5, and 6), or one to three months after the second IVIg course (patients 1, 2, and 3) and was subsequently adjusted (range $0.5-3 \mathrm{mg} / \mathrm{kg} /$ day) to maintain the white blood cell count between 3000 and $3500 / \mathrm{mm}^{3}$. To decrease the urothelial toxicity of CTX, all patients were given 300-600 mg oral $\mathrm{N}$-acetyl cysteine/day while on the drug. ${ }^{18}$

CLINICAL ASSESSMENT

All patients were assessed before treatment, before every two day IVIg infusion, and every six months after stopping treatment using (a) a Medical Research Council (MRC) rating scale to measure muscle strength in the 10 most affected muscles of the upper and lower limbs (total 20 muscles, maximal score $=100$ ); (b) a modified Rankin disability scale ${ }^{9}(0=$ asymptomatic, $1=$ non-disabling symptoms not interfering with lifestyle, $2=$ minor disability symptoms leading to some restriction of lifestyle but not interfering with the patients' capacity to look after themselves, $3=$ moderate disability symptoms significantly interfering with lifestyle or preventing fully independent existence, $4=$ moderately severe disability symptoms preventing independent existence although patients do not need constant attention day and night, $5=$ totally dependent, requiring constant attention day and night; (c) a functional impairment scale for upper and lower limbs ${ }^{9}$ $(0=$ asymptomatic, $1=$ upper and lower limb symptoms without functional impairment (normal walk), 2=some minor difficulties in manual activities/abnormal walk without support, $3=$ unable to perform some manual activities/independent walking with support, $4=$ not capable of manual tasks/needing help to walk, $5=$ total paralysis/confined to a wheelchair.

\section{NERVE CONDUCTION STUDIES}

Nerve conduction studies were performed in all patients before treatment, at six and 12 months, then yearly during the follow up. Motor conduction studies were performed in the ulnar, median, peroneal, posterior tibialis, and radial nerves using surface electrodes. ${ }^{9}$ The focality of partial motor conduction block (see above) was confirmed, when possible, by the repeated stimulation of nerves at several sites as close as $2.5 \mathrm{~cm}$ apart. Sensory conduction studies were performed in the median, ulnar, and sural nerves.

ANTI-GM1, ASIALOGM1, AND GD1a ANTIBODIES Anti-GM1, asialoGM1, and GD1a antibodies were measured by ELISA before treatment and yearly during the follow up, but before IVIg maintenance infusions, using a previously reported procedure, ${ }^{20}$ except that detergent was omitted in all solutions, and that all incubations were carried out at $4^{\circ} \mathrm{C}$.

\section{Results}

The table shows the clinical, electrophysiological, and immunological findings before and after therapy and the duration of treatment and of follow up in the six patients. All patients improved during treatment. By the end of follow up (mean 47, range 37-61 months), or before relapse after stopping treatment (patients 1, 2, 3, and 4), the median Rankin $(\mathrm{P}=0.0335)$ and upper limb $(t=6.325$; $\mathrm{P}=0.0015)$ and lower limb $(t=3.0 ; \mathrm{p}=0.0301)$ impairment scores and the mean MRC score $(\mathrm{P}=0.0561)$ were improved (table). By that time the number of nerves with partial motor conduction block was significantly reduced $(\mathrm{P}=0.0197)$ (table). The reduction of partial motor conduction block ranged from $15 \%$ to $87 \%$ (mean $45 \%$ ) in the 12 "improved" nerves at the end of follow up and from $10 \%$ to $31 \%$ (mean 21\%) in the three nerves in which partial motor conduction block was still present at the end of follow up whereas partial motor conduction block became evident in an

Long term effect of IVIg and CTX treatment in patients with multifocal motor neuropathy

\begin{tabular}{|c|c|c|c|c|c|c|c|c|c|c|c|c|c|}
\hline \multirow[b]{2}{*}{$\begin{array}{l}\text { Patient } \\
\text { No }\end{array}$} & \multirow[b]{2}{*}{ Age/Sex } & \multirow{2}{*}{$\begin{array}{l}\text { Duration } \\
\text { before } \\
\text { treatment }\end{array}$} & \multicolumn{2}{|c|}{$\begin{array}{l}\text { Months of } \\
\text { treatment }\end{array}$} & \multirow{2}{*}{$\begin{array}{l}\text { Total } \\
\text { months of } \\
\text { follow up }\end{array}$} & \multicolumn{2}{|c|}{ MRC score * } & \multicolumn{2}{|c|}{ Rankin score } & \multicolumn{2}{|c|}{ Nerves with $P M C B$} & \multicolumn{2}{|c|}{ Antiglycolipid titrest } \\
\hline & & & $I V I g$ & $C T X$ & & $\begin{array}{l}\text { Before } \\
\text { treatment }\end{array}$ & $\begin{array}{l}\text { At last } \\
\text { follow up }\end{array}$ & $\begin{array}{l}\text { Before } \\
\text { treatment }\end{array}$ & $\begin{array}{l}\text { At last } \\
\text { follow up }\end{array}$ & $\begin{array}{l}\text { Before } \\
\text { treatment }\end{array}$ & $\begin{array}{l}\text { At last } \\
\text { follow up }\end{array}$ & Before treatment & $\begin{array}{l}\text { At last } \\
\text { follow up }\end{array}$ \\
\hline 1 & $48 / \mathrm{M}$ & $10 \mathrm{y}$ & $1 \rightarrow 13$ & $3 \rightarrow 25$ & 55 & 66 & $83 \ddagger \rightarrow 77 \S$ & 2 & 1 & 2 & $1 \neq \rightarrow 2 \S$ & 640 (GM1) & Neg $\neq 0$ \\
\hline 2 & $26 / \mathrm{F}$ & $5 \mathrm{y}$ & $1 \rightarrow 44$ & $5 \rightarrow 44$ & 61 & 37 & $83 \ddagger \rightarrow 81 \oint$ & 4 & 1 & 4 & $0 \ddagger \rightarrow 1 \rrbracket$ & Neg & Neg \\
\hline 3 & $51 / \mathrm{F}$ & $4 \mathrm{y}$ & $1 \rightarrow 36$ & $3 \rightarrow 45$ & 45 & 81 & $85 \ddagger \rightarrow 76 ₫$ & 2 & $1 \ddagger \rightarrow 2 \varsigma$ & 3 & $1 \neq \rightarrow 2 \int$ & 40960 (aGM1) & $40960 \neq \$$ \\
\hline 4 & $29 / \mathrm{M}$ & 3 months & $1 \rightarrow 9$ & $1 \rightarrow 13$ & 46 & 89 & $95 \ddagger \rightarrow 89 \rrbracket$ & 2 & $1 \ddagger \rightarrow 2 \coprod$ & 2 & $0 \ddagger \rightarrow 2 \coprod$ & $\varnothing 2560(\mathrm{GD} 1 \mathrm{a})$ & Neg $\neq \varnothing$ \\
\hline 5 & $34 / \mathrm{M}$ & $4 \mathrm{y}$ & $1 \rightarrow 37$ & $1 \rightarrow 37$ & 37 & 79 & 86 & 2 & 2 & 2 & 2 & $\varnothing 5120$ (GD1a) & 2560 \\
\hline 6 & $60 / \mathrm{F}$ & $10 \mathrm{y}$ & $1 \rightarrow 37$ & $1 \rightarrow 37$ & 37 & 65 & 80 & 3 & 2 & 2 & 0 & Neg & Neg \\
\hline Mean & & & 29.3 & 31.7 & 46.8 & 69.5 & $85.3 \ddagger$ & & & & & & \\
\hline Median & & & & & & & & 2 & $1 \ddagger \|$ & & & & \\
\hline Total & & & & & & & & & & 15 & $4 \ddagger^{\star \star}$ & & \\
\hline
\end{tabular}

${ }^{\star}$ Calculated in the 20 more severely affected muscles.

†IgM titres (inverse of serum dilution) except $\varnothing$ (IgG). Normal upper limits: GM=1/320; asialoGM1 (aGM1)=1/5120; GD1a=1/640.

$\ddagger$ At last follow up before relapse.

At relapse, after the end of treatment.

I $t=-2.476, \mathrm{P}=0.0561 ; \| t=2.907, \mathrm{P}=0.0335 .{ }^{\star \star} t=3.379, \mathrm{P}=0.0197$ (Student's $t$ test for paired data).

$\mathrm{PMCB}=$ partial motor conduction block. 

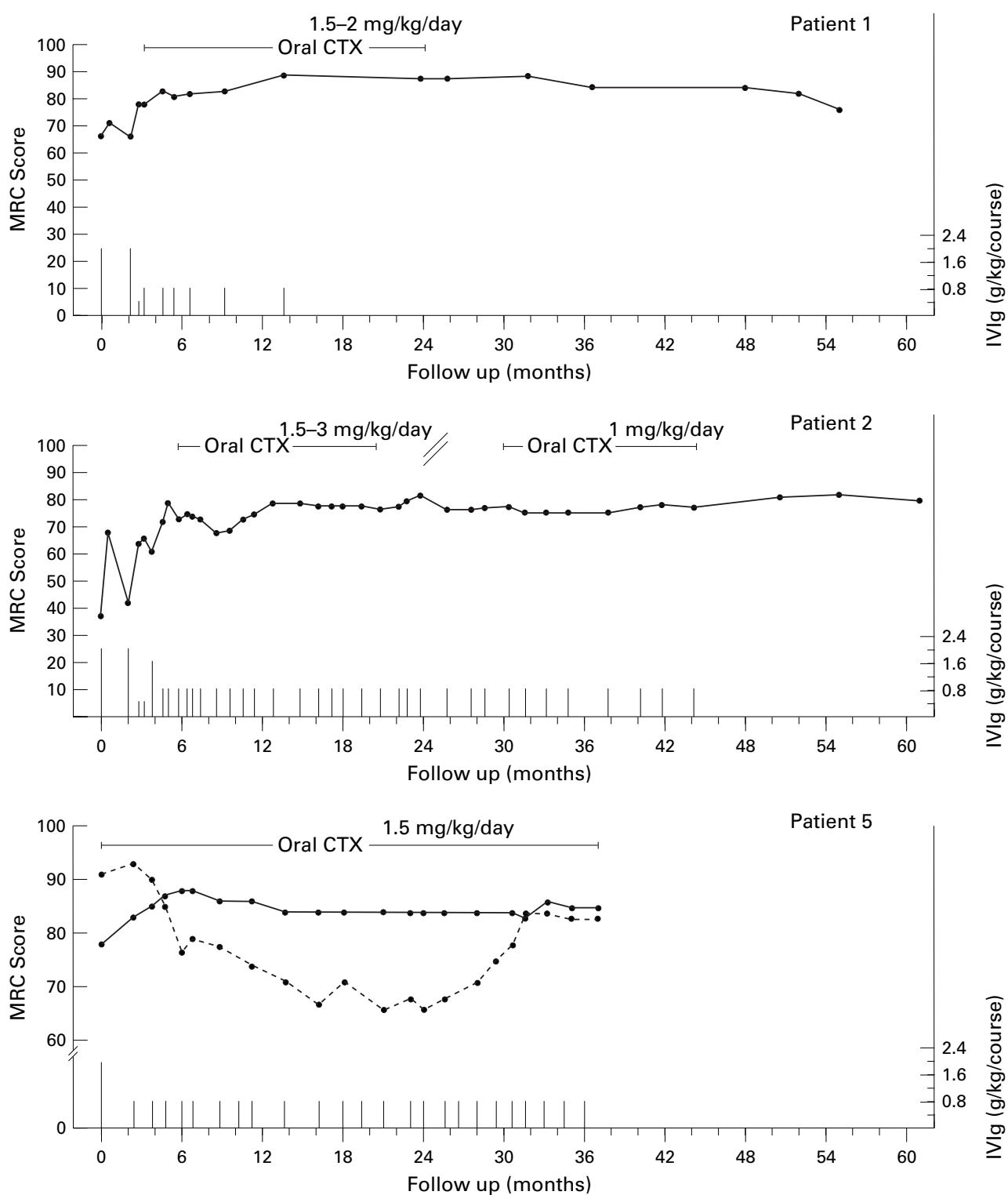

Clinical response to long term treatment with high dose intravenous immunoglobulins (IVIg) and oral cyclophosphamide (CTX), expressed as MRC score (the sum of the strength in the 10 most affected muscles in the upper and lower limbs, total 20 muscles, maximum score 100) in three representative patients $(1,2$, and 5) with multifocal motor neuropathy. The vertical bars indicate IVIg courses and their total dosage (see scale on the right). In patients 1 and 2, the intervals between periodic worsening and necessary IVIg infusions became progressively longer after three and seven months respectively of oral CTX, and treatment could be stopped for 24 and 17 months respectively, before clinical worsening. Infusions of IVIg could not be suspended after the addition of oral CTX in patient 5 who improved in the most affected muscles (continuous line) while receiving two day IVIg courses every 60 days but worsened in other muscles (broken lines). The deterioration was reversed by increasing the frequency of IVIg infusions.

additional nerve in one patient (5). Within 12 to 24 months of CTX, titres of antiglycolipid antibodies progressively decreased in three of four patients, whereas they remained unchanged in one patient throughout the follow up (table). A clinical relapse, incurred after temporary suspension of therapy, was always associated with an increase in the number of nerves with partial motor conduction block but not with a rise in antiglycolipid titres (table).

In all patients the effect of IVIg initially lasted 20-30 days and improvement had to be maintained with periodic IVIg infusions (figure). After three to seven months of oral CTX the intervals between periods of worsening and necessary IVIg infusions became progressively longer. The mean monthly dose of IVIg decreased from $91.1 \mathrm{~g} /$ patient during the first three months (during which one or (in three patients), two five day courses of IVIg were performed) to $57.9 \mathrm{~g}$ during the second three months, and to $32-34 \mathrm{~g}$ during the second six months and, in those treated longer, during the second and third year. In three patients $(1,2$, and 4) IVIg infusions were suspended after 13, 44 , and nine months respectively, when improvement became stable, although two continued CTX for other 12 (patient 1) and four (patient 4) months (figure). Two of these patients (1 and 4) worsened two years after suspending all therapies whereas the other (patient 2) started to deteriorate 17 months 
after stopping treatment. They are now improving again with IVIg. The other three patients $(3,5$, and 6$)$ still require periodic IVIg infusions after three years of IVIg and CTX. In two patients (3 and 6) improvement is reinstated with two day IVIg courses every 60-70 days when worsening is seen. This worsening was most evident in patient 3 who stopped IVIg for nine months while continuing with oral CTX. Patient 5 improved in the most affected muscles while receiving two day IVIg treatments every 60 days but, at the same time, he worsened in other muscles, as has been recently reported. ${ }^{21}$ The deterioration was reversed by increasing IVIg infusions to every 30-40 days (figure).

At the end of the first IVIg infusions, four patients developed mild transient headache, associated in three with nausea and moderate fever and, in two, with itching. These symptoms were controlled with noramidopiridine and were subsequently prevented by the prescription of chlorpheniramine maleate. No other adverse events were reported for IVIg therapy. Serum creatinine concentrations at the end of follow up were increased by less than $10 \%$ of the pretreatment value in four patients, by $20 \%$ (from 0.9 to $1.08 \mathrm{mg} / \mathrm{dl}$, upper normal limit $1.2 \mathrm{mg} / \mathrm{dl}$ ) in one (patient 4 ), and by nearly $30 \%$ (from 0.7 to 0.9 ) in one (patient 2). Two patients developed haemorrhagic cystitis after 15 and 13 months of CTX, at mean daily doses of 125 and $150 \mathrm{mg} /$ day. This complication promptly resolved in both patients after stopping CTX. One of these patients also developed persistent amenorrhea after one year of CTX. No complications related to CTX were found in the other patients, who required a lower mean daily dose of oral CTX (from 25 to $90 \mathrm{mg} /$ day) to induce leucopenia.

\section{Discussion}

We treated six patients with multifocal motor neuropathy with periodic IVIg infusions and oral CTX for 37 to 61 (mean 47) months. By the end of follow up or until adequately treated, all patients were clinically better than before therapy. In all patients improvement correlated with a reduction in partial motor conduction block, as previously found in patients with multifocal motor neuropathy treated with IVIg. ${ }^{92}{ }^{23}$ Antiglycolipid titres were also reduced in three of the four patients who tested positively although they did not rise at times of relapse. A similar reduction in antibody titres was previously reported in patients with multifocal motor neuropathy treated with $\mathrm{CTX}^{78}$ but not with IVIg, ${ }^{9}$ suggesting that CTX but not IVIg might reduce antibody synthesis in these patients.

In all patients periodic IVIg infusions were initially necessary to maintain improvement. This is in accordance with previous studies, ${ }^{9-14}$ which showed that the effect of IVIg in most patients with multifocal motor neuropathy lasts only a few weeks and has to be maintained with periodic infusions for a long time, if not indefinitely, ${ }^{15-17}$ suggesting that IVIg may induce and maintain improvement but that it does not eradicate the disease. ${ }^{21}$ The addition of oral CTX was followed in all our patients, within three to seven months, by a reduction of required IVIg infusions and, in three patients, all therapies could be suspended for close to two years. The proportion of patients achieving a sustained remission with this combination was greater than that reported with IVIg alone. ${ }^{12}{ }^{15-17}$ This suggests that oral CTX may help to induce remission in this disease, as was indicated by the improvement with a similar time delay in patients with multifocal motor neuropathy treated with high dose intravenous CTX. ${ }^{78}$ However, as multifocal motor neuropathy may have a stepwise progression ${ }^{5}$ and, occasionally, show spontaneous recovery, ${ }^{3}$ it is not possible to exclude that a remission reflects, at least in some patients, the natural course of the disease. This might also explain the long term benefit of IVIg in some patients with multifocal motor neuropathy treated with IVIg alone, ${ }^{912}$ even if the duration of this sustained effect is not known. These findings confirm that IVIg infusions need to be tailored for each patient to avoid unnecessarily protracted treatments during remission.

Although oral CTX may be useful for delaying IVIg infusions and, possibly, for inducing a sustained remission, it is not devoid of side effects. This drug, especially when taken at high dosages or for long periods, has been associated with life threatening complications, including hepatic injury, opportunistic infections, and remote development of malignancies. ${ }^{18} 19$ The relative risk-benefit of combining CTX with IVIg for the treatment of multifocal motor neuropathy requires further investigation in a controlled study. Until then it might be appropriate to reserve oral CTX for patients with multifocal motor neuropathy who continue to require frequent IVIg infusions for several months to maintain clinical improvement.

We thank Mrs Silvia Allaria from our Institute for her valuable technical assistance. This work was supported by Associazione Amici Centro Dino Ferrari, Telethon, Italy (grant No 674), Associazione Italiana Sclerosi Multipla, Italian National Regretto finalizzato Neuroimmunoligia), and IRCCS Ospedale Maggiore Policlinico, Milan, Italy.

1 Parry GH, Clarke S. Multifocal acquired demyelinating neuropathy masquerading as motor neuron disease. Muscle Nerve 1988;11:103-7.

2 Roth G, Rohr J, Magistris MR, Ochsner F. Motor neuropathy with proximal multifocal persistent conduction block, fasciculations, and myokymia. Eur Neurol 1986;25: 416-23.

3 Chad DA, Hammer K, Sargent J. Slow resolution of multifocal weakness and fasciculation: a reversible motor neuron syndrome. Neurology 1986;36:1260-3.

4 Kornberg AJ, Pestronk A. Chronic motor neuropathies: diagnosis, therapy and pathogenesis. Ann Neurol 1995;37:
diand diagnosis,

5 Nobile-Orazio E. Multifocal motor neuropathy [editorial]. $\mathcal{F}$ Neurol Neurosurg Psychiatry 1996;60:599-603.

6 Lange DJ, Trojaburg W, Latov N, et al. Multifocal motor neuropathy with conduction block: is it a distinct clinical entity? Neurology 1992;42:497-505.

7 Pestronk A, Cornblath DR, Ilyas A, et al. A treatable multifocal motor neuropathy with antibodies to GM1 ganglioside. Ann Neurol 1988;24:73-8.

8 Feldman EL, Bromberg MB, Albers JW, Pestronk A. Immunosuppressive treatment in multifocal motor neuropathy. Ann Neurol 1991;30:397-401.

9 Nobile-Orazio E, Meucci N, Barbieri S, Carpo M, Scarlato G. High dose intravenous immunoglobulin therapy in multifocal motor neuropathy. Neurology 1993;43:537-44.

10 Chaudhry V, Corse A, Cornblath DR, et al. Multifocal motor neuropathy: response to human immune globulin. Ann Neurol 1993;33:237-42. 
11 Azulay JP, Blin O, Pouget J, et al. Intravenous immunoglobulin treatment in patients with motor neuron syndromes associated with anti-GM1 antibodies. Neurology 1994;44: 429-32.

12 Bouche P, Moulonguet A, Younes-Chennoufi AB, et al. Multifocal motor neuropathy with conduction block: a study of 24 patients. F Neurol Neurosurg Psychiatry 1995;59: $38-44$

13 Van den Berg LH, Kerkhoff H, Oey PL, et al. Treatment of multifocal motor neuropathy with high dose intravenous immunoglobulins: a double blind, placebo controlled study. $\mathcal{F}$ Neurol Neurosurg Psychiatry 1995;59: 248-52.

14 Kaji R, Shibasaki H, Kimura J. Multifocal demyelinating motor neuropathy: cranial nerve involvement and immunoglobulin therapy. Neurology 1992;42:506-9.

15 Azulay JP, Rihet P, Pouget J, Cador A, Crevat A, Serratrice G. Long-term therapy of multifocal motor neuropathy: Report of 18 patients [abstract]. Neurology 1995;45(suppl R):A416-7.

16 Van den Berg LH, Franssen H, Wokke JHJ. Improvement of multifocal motor neuropathy during long-term weekly treatment with human immunoglobulin. Neurology 1995 45:987-8.
17 Wokke JHJ, Van den Berg LH, Franssen H, Oey PL. Longterm effect of intravenous immunoglobulin treatment in patients with multifocal motor neuropathy [abstract]. Neurology 1996;46(suppl):A381.

18 Levine LA, Riche JP. Urological complications of cyclophospahmide. F Urol 1989;141:1063-9.

9 Calabresi P, Chabner BA. Antineoplastic agents. In: Goodman Gilman A, Rall TW, Nies AS, Taylor P, eds. The pharmacological basis of therapeutics. 8th ed. New York: McGraw-Hill, 1991:1209-63.

20 Nobile-Orazio E, Carpo M, Legname G, Meucci N, Sonnino S, Scarlato G. Anti-GM1 IgM antibodies in motor neuron disease and neuropathy. Neurology 1990;40:174750.

21 Elliot JL, Pestronk A. Progression of multifocal motor neuropathy during apparently successful treatment with human immunoglobulin. Neurology 1994;44:967-8.

22 Chaudhry V, Corse AM, Cornblath DR, Kuncl RW, Freimer ML, Griffin JW. Multifocal motor neuropathy: Freimer ML, Griffin JW. Multifocal motor neuropathy:

23 Cappellari A, Nobile-Orazio E, Meucci N, Scarlato G, Barbieri S. Multifocal motor neuropathy: a possible source of error in the serial evaluation of conduction block. Muscle Nerve 1996;19:666-9. 